\title{
Synchronous primary malignant neoplasms of the cervix and endometrium
}

\author{
SHULAN LV ${ }^{1 *}$, XUE XUE $^{1 *}$, YANXIA SUI ${ }^{2}$, JUANG DU $^{1}$, JUNKAI ZOU $^{1}$, \\ CHAO SUN ${ }^{1}$, DONG LIU ${ }^{1}$, QING SONG ${ }^{1,3}$ and QILING LI ${ }^{1}$ \\ Departments of ${ }^{1}$ Obstetrics and Gynecology and ${ }^{2}$ Pathology, First Affiliated Hospital, Xi'an Jiaotong University, Xi'an, \\ Shaanxi 710061, P.R. China; ${ }^{3}$ Cardiovascular Research Institute, Morehouse School of Medicine, Atlanta, GA 30310, USA
}

Received April 24, 2015; Accepted February 28, 2017

DOI: $10.3892 / \mathrm{mco} .2017 .1202$

\begin{abstract}
Synchronous multiple malignant neoplasms of the female genital tract are rare, particularly in the uterus. We herein present the case of a patient with synchronous cervical squamous epithelial carcinoma and endometrial adenocarcinoma, and discuss the diagnosis, treatment and prognosis. The patient underwent a cervical biopsy and fractional curettage of the endometrium, followed by abdominal staging surgery, including radical hysterectomy, bilateral salpingo-oophorectomy, pelvic and para-aortic lymphadenectomy. The diagnosis was stage Ib1 cervical cancer and stage Ia endometrial cancer, without lymph node metastases. There has been no recurrence during 1 year of followup. Synchronous genital tract neoplasms are more clinically complex compared with single neoplasms and it is crucial to focus on the differential diagnosis between primary and metastatic tumors during the diagnostic process. The treatment of synchronous genital tract neoplasms also differs significantly from that of single neoplasms, although the prognosis of patients with synchronous gynecological malignancies does not appear to be worse.
\end{abstract}

\section{Introduction}

Synchronous primary genital cancer is an uncommon occurrence. In a retrospective study of 3,863 patients with female genital malignancies, $26(0.7 \%)$ patients with synchronous primaries were identified (1). The concomitant malignancies are frequently diagnosed as metastatic disease. In the majority of the cases, the patients with $>1$ gynecological neoplasms present with synchronous endometrial and ovarian cancer (1),

Correspondence to: Professor Qiling Li, Department of Obstetrics and Gynecology, First Affiliated Hospital, Xi'an Jiaotong University, 277 Yanta West Road, Xi'an, Shaanxi 710061, P.R. China

E-mail: liqiling@mail.xjtu.edu.cn

*Contributed equally

Key words: cervical cancer, endometrial cancer, synchronous primary malignancies whereas synchronous primary malignancies of the uterus are even rarer $(2,3)$. We herein present an unusual case of cervical squamous cell carcinoma and endometrial adenocarcinoma occurring simultaneously. The aim of this case report was to evaluate the diagnosis and treatment of synchronous cancers of the cervix and endometrium.

\section{Case report}

A 48-year-old Chinese female patient (body mass index, $26.8 \mathrm{~kg} / \mathrm{m}^{2}$, gravida 2, para 2), who had a history of a sterilization operation, presented in July, 2013 at the First Affiliated Hospital of Xi'an Jiaotong University (Xi'an, China) with irregular vaginal spotting for 5 months. The patient's last menstrual period was on July 7, 2013. Other past history and family history were unremarkable. After attending a regional hospital for spotted vaginal bleeding, the patient underwent a cervical biopsy during colposcopy due to a $0.3-\mathrm{cm}$ cauliflower-like lesion in the anterior cervical lip, and fractional curettage of the endometrium. The pathological examination of the bioptic material in our pathology department revealed a poorly differentiated squamous cell carcinoma in the cervix (Fig. 1) and a poorly differentiated adenocarcinoma in the uterine cavity (Fig. 2). Physical examination in our hospital revealed a soft abdomen, a minor ulcer-like lesion in the anterior cervical lip with a diameter of $0.5 \mathrm{~cm}$, and an enlarged uterus, similar to the 11th gestational week. Pelvic examination revealed a movable cervix with a grossly smooth appearance, well-supported smooth vagina, free parametrium and movable uterosacral ligaments. Magnetic resonance imaging of the pelvic cavity revealed an abnormal signal in the anterior lip of the cervix and a $5 \times 4 \times 1-\mathrm{cm}$ thickening of the endometrium in the uterine cavity involving the upper part of the corpus. The preoperative serum tumor marker levels were as follows: Carbohydrate antigen (CA) 125, $15.35 \mathrm{U} / \mathrm{ml}$ (normal range, 0-35 U/ml), CA 19-9, $5.16 \mathrm{ng} / \mathrm{ml}$ (normal range, 0-40 ng/ml) and carcinoembryonic antigen, $1.48 \mathrm{ng} / \mathrm{ml}$ (normal range, $\leq 5 \mathrm{ng} / \mathrm{ml}$ ). The patient had never participated in a cervical screening procedure, and the human papillomavirus (HPV) test results revealed infection by type 18 .

As the cervical cancer was at clinical stage Ib1 and the endometrial cancer was at stage Ia according to the International Federation of Gynecology and Obstetrics (FIGO) classification, the patient underwent abdominal staging surgery, including 

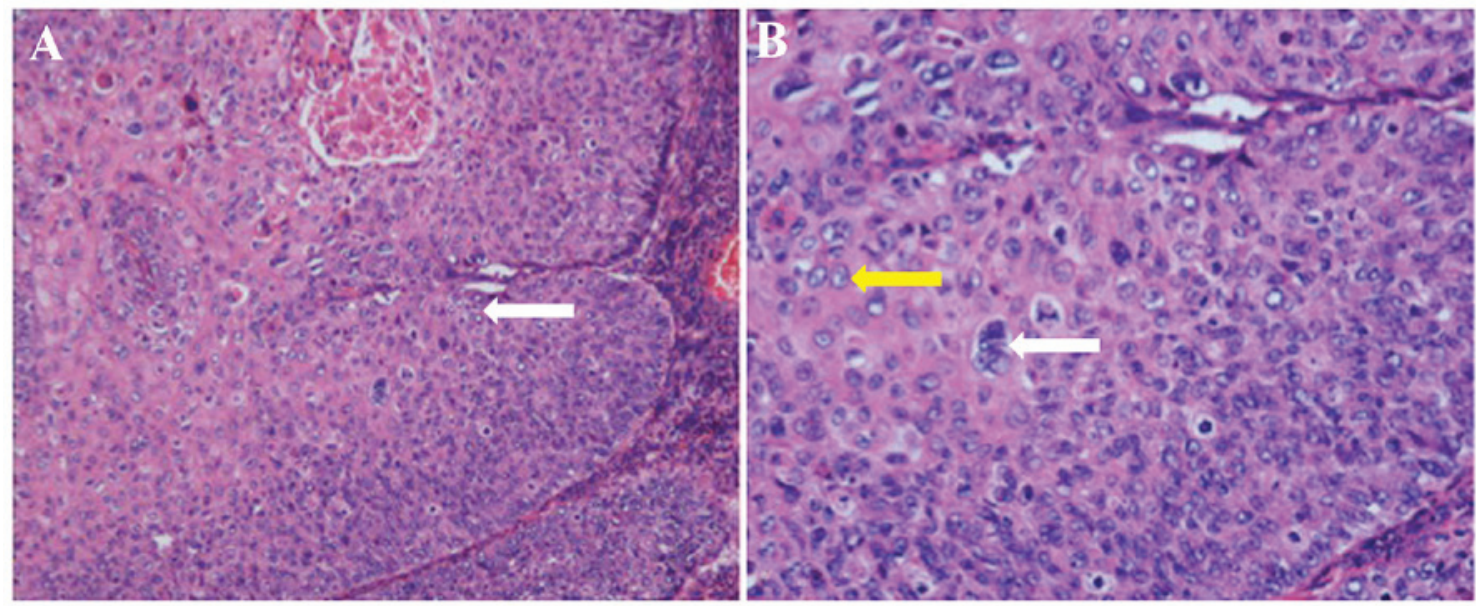

Figure 1. Squamous carcinoma of the cervix. (A) Squamous carcinoma cells were arranged in sheets or clusters (arrow) [hematoxylin and eosin staining (H\&E); magnification, x100]. (B) The cytoplasm of the tumor cells was eosinophilic. Cells with vesicular nuclei (yellow arrow) and megakaryocytes (white arrow) were observed (H\&E; magnification, $\mathrm{x} 400)$.
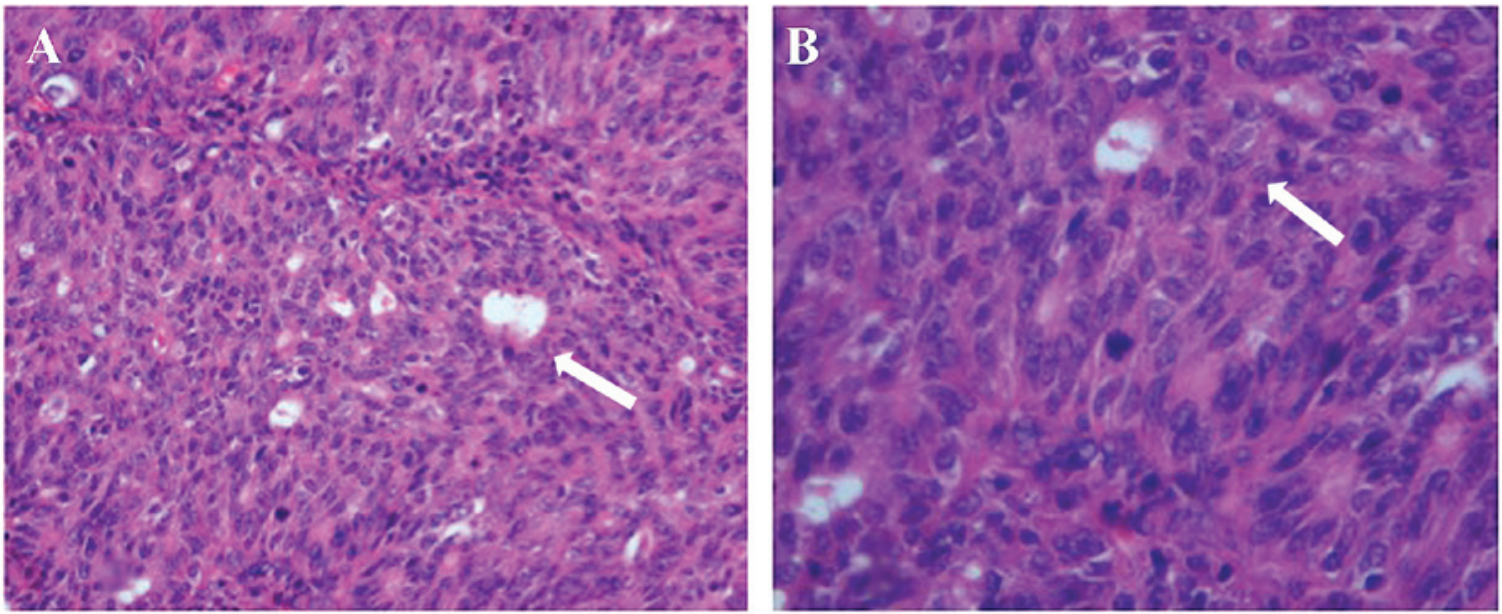

Figure 2. Adenocarcinoma of the endometrium. (A) Adenocarcinoma cells in acinar formations (arrow) [hematoxylin and eosin staining (H\&E); magnification, x200]. (B) The cell boundaries were indistinct, with a high nucleus:cytoplasm ratio. There was significant cellular atypia (arrow) and mitotic figures (H\&E; magnification, $\mathrm{x} 400$ ).

radical hysterectomy, bilateral salpingo-oophorectomy, pelvic and para-aortic lymphadenectomy. Macroscopically, the uterus was enlarged, measuring $12 \times 8 \times 6 \mathrm{~cm}^{3}$, with a minor ulcer-like lesion in the anterior lip of the cervix measuring $0.5 \mathrm{~cm}$ in greatest diameter. The adnexa and parametrium were unremarkable. A fungating tumor, sized $5 \times 4.5 \times 1 \mathrm{~cm}^{3}$, was identified in the uterine cavity, with superficial invasion of the myometrium. Hematoxylin and eosin-stained sections revealed poorly differentiated endometrial adenocarcinoma with superficial myometrial invasion $(<50 \%)$ in the corpus uteri. No residual carcinoma cells were found in the resection margin of the vagina, and lymph node mapping revealed no metastasis. There was chronic cervicitis with local squamous metaplasia of the cervix and Naboth's cysts, bilateral hydrosalpinx and Graafian follicles in the bilateral ovaries. The results of the immunohistochemical examination of the carcinomas of the cervix and endometrium were as follows: Creatine kinase ${ }^{+}$ (Fig. 3A), P16 ${ }^{+}$(Fig. 3B), vimentin ${ }^{+}$(Fig. 3C), human epidermal growth factor receptor- $2^{+}\left(2^{+}\right)$(Fig. 3D), estrogen receptor ${ }^{+}\left(2^{+}\right.$,
90\%) (Fig. 4A), progesterone receptor ${ }^{+}\left(3^{+}, 80 \%\right)$ (Fig. 4B), Ki-67 $7^{+}(80 \%)$ (Fig. 4C), P53 ${ }^{+}(80 \%)$ (Fig. 4D), cytokeratin (CK) $5 / 6^{-}, \mathrm{P}^{-} 3^{-}, \mathrm{P}^{-} 0^{-}$and $\mathrm{CK} 14^{-}$. The patient was thoroughly evaluated and the FIGO stages were cervical cancer stage Ib1 and endometrial cancer stage Ia, without lymph node metastases. The patient is currently followed up every 3 months. The date of the last follow-up was May 24, 2016 and no recurrence was observed.

\section{Discussion}

Synchronous tumors are defined as two or more tumors occurring in a patient simultaneously. In 1879, Billroth and Hackley were the first to report the development of different primary malignant tumors in the same patient (4). Multiple primary cancers are an infrequent finding. The etiology and pathogenesis of synchronous cancers remain unclear. When similar tissues of the female genital tract are subjected to carcinogen exposure during fetal/embryological development, 

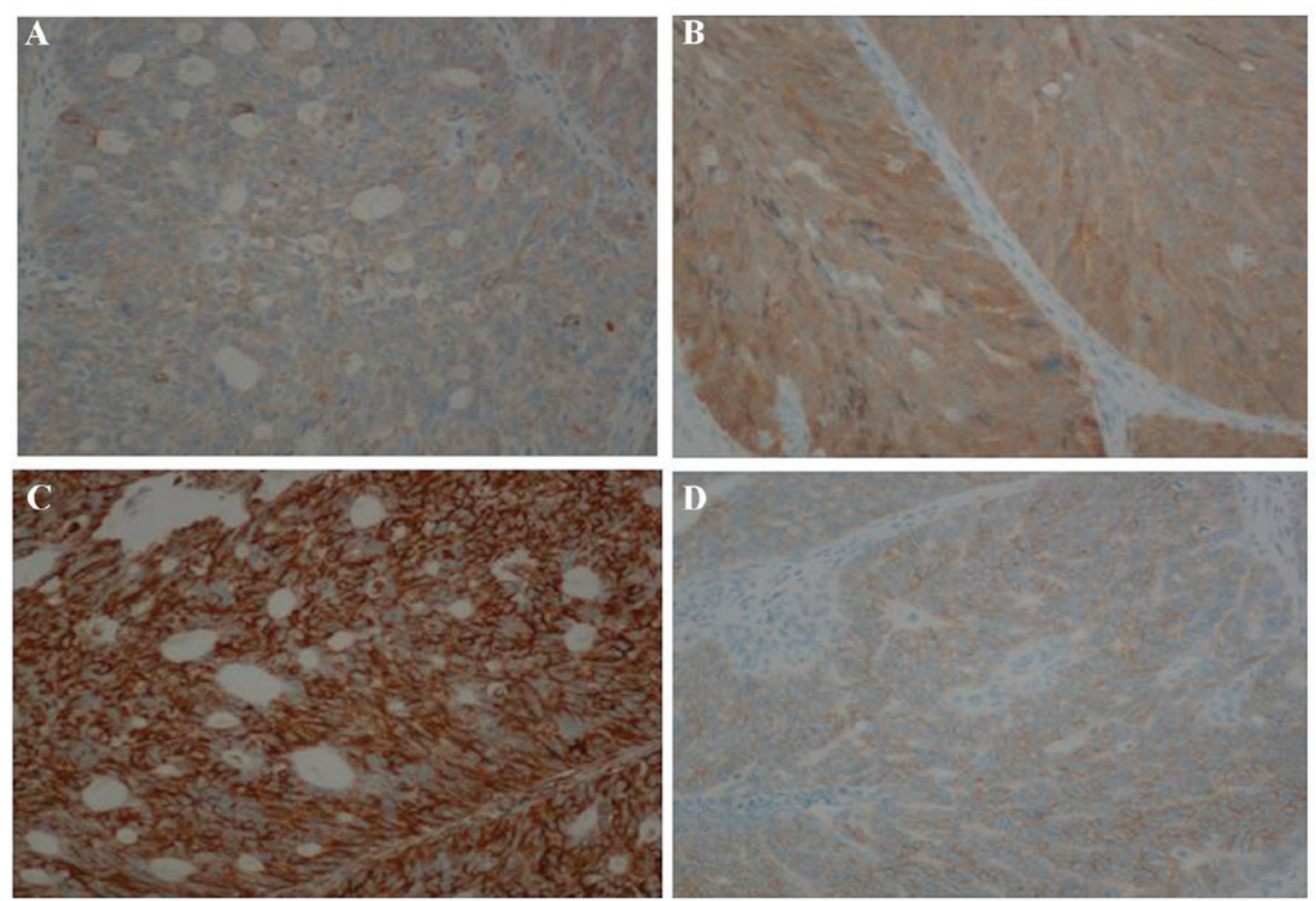

Figure 3. (A) Immunohistochemical staining of cervix for P16; Immunohistochemical staining of the endometrium for (B) creatine kinase and (C) vimentin revealed cytoplasmic positivity confined to the glands (original magnification, x400). (D) Immunohistochemical staining for human epidermal growth factor receptor- $2\left(2^{+}\right)$revealed cell membrane positivity confined to the glands.
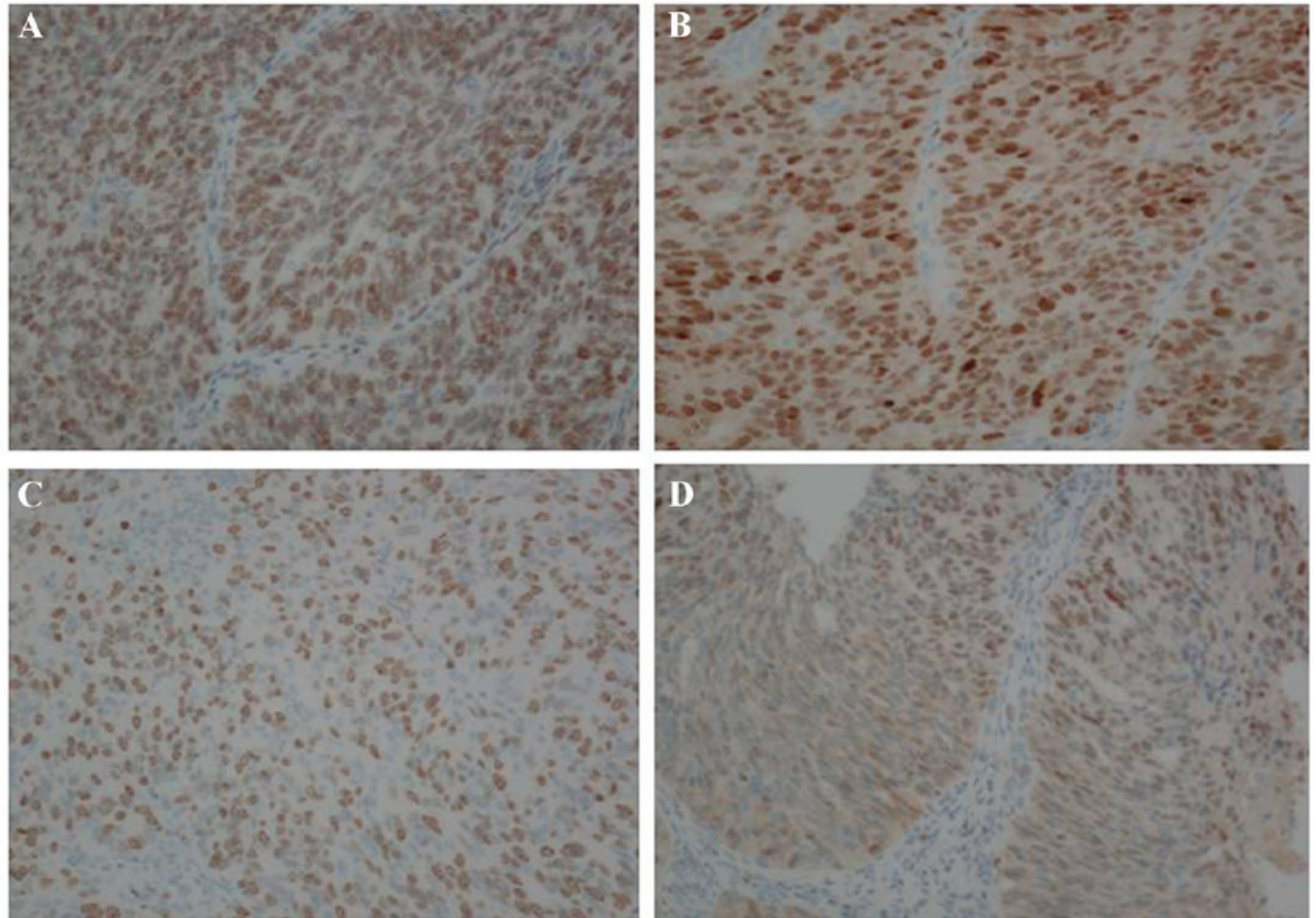

Figure 4. Immunohistochemical staining of the endometrium for (A) estrogen receptor (2+, 90\%), (B) progesterone receptor (3+, $80 \%)$, (C) Ki-67 (80\%) and (D) $\mathrm{P}^{+} 3^{+}(80 \%)$ revealed nuclear positivity confined to the glands (original magnification, $\mathrm{x} 400$ ).

synchronous tumors may develop (5). In gynecological oncology, endometrial cancers with simultaneous ovarian cancers are the most common, whereas, to the best of our knowledge, only 9 cases of carcinoma of the cervix with 
carcinoma of the endometrium have been reported to date (3,6-10); the tumors comprised different histopathological subtypes, whereas in only two cases $(\sim 22 \%)$ the types were the same as in the present case.

Unlike the single aggressive ovarian cancers that usually remain asymptomatic until they reach an advanced stage, endometrial and cervical cancers usually produce earlier symptoms, such as abnormal vaginal bleeding. Therefore, the disease was stage I at diagnosis in the present case. The diagnosis of synchronous cancers generally relies on postoperative pathological examination, as the same pathological patterns may be considered as metastatic carcinoma. In the present case, the diagnosis of synchronous cancers was made prior to surgery, as the cancers were of different pathological type. In order to improve the accuracy of diagnosis of synchronous cervical and endometrial cancers, when contact bleeding is observed, the cervix and endometrium should be more closely inspected. Fractional curettage may be performed if necessary. When the diagnosis of endometrial cancer is confirmed, a Pap smear or ThinPrep cytological test should be performed to exclude cervical cancer. Biopsy is of the same diagnostic value as curettage in patients with endometrial cancer, particularly those with cancer of the cervical canal. Stage should be thoroughly evaluated pre- and postoperatively. Staging should be performed in combination with clinicopathological diagnosis in order to determine the treatment strategy. At present, none of the available staging systems is considered to be suitable and a new system of staging and treatment determination must be established. According to the results of diagnostic dilatation and curettage and colposcopic biopsy, some of the cases may be diagnosed preoperatively and the precise extent of the resection may be determined.

Radical hysterectomy is the recommended treatment for stage Ib1 cervical cancer. During the operation, resection of the sacral and main ligaments and vaginal and pelvic lymphadenectomy are crucial. For stage Ia poorly differentiated endometrial adenocarcinoma, hysterectomy may be sufficient, provided bilateral adnexectomy, pelvic and para-aortic lymphadenectomy are performed. For our patient, these two types of operations had to be combined. Radical hysterectomy with bilateral adnexectomy, pelvic and para-aortic lymphadenectomy were performed. Adjuvant concurrent chemoradiotherapy was deemed necessary based on postoperative FIGO staging. The treatment strategies for synchronous cancers depend on the established strategy for each individual cancer and, occasionally, both tumors may be treated simultaneously.

The prognosis of patients with synchronous primary cervical and endometrial cancers is associated with disease stage at the time of diagnosis. In general, synchronous cancers are often considered worse when compared with malignancies of a metachronous nature, but are associated with significantly higher survival rates compared with metastatic cases $(5,7,10)$. In every follow-up visit, an HPV text of the vaginal stump or surgical scar should be performed. As endometrial cancer may metastasize to the lungs, a chest X-ray is also reasonable when necessary. Standard therapy including surgery and/or radiation therapy is strongly recommended. In conclusion, the occurrence of synchronous primary gynecological cancers is an uncommon occurrence deserving further investigation.

This case report has been approved by the Institutional Review Board of the First Affiliated Hospital of Xi'an Jiaotong University. Written informed consent was obtained from the patient for publication of the details of this case and any accompanying images.

\section{References}

1. Eifel P, Hendrickson M, Ross J, Ballon S, Martinez A and Kempson R: Simultaneous presentation of carcinoma involving the ovary and the uterine corpus. Cancer 50: 163-170, 1982.

2. Sun HD, Lai CR, Yen MS and Wang PH: Synchronous occurrence of primary neoplasms of the uterus with mucinous carcinoma of the cervix and endometrioid carcinoma of the endometrium. Taiwan J Obstet Gynecol 50: 377-378, 2011.

3. Hascalik S, Celik O, Erdem G, Usta U, Alkan A, Karakas HM and Mizrak B: Synchronous endometrial and cervical tumors in a 26-year-old nullipara: Diagnostic modalities updated. Gynecol Obstet Invest 60: 171-176, 2005.

4. Billroth $T$ and Hackley CE: General surgical pathology and therapeutics. D. Appleton and company, New York, 1879.

5. Eisner RF, Nieberg RK and Berek JS: Synchronous primary neoplasms of the female reproductive tract. Gynecol Oncol 33: 335-339, 1989.

6. Lin CK, Yu MH, Chu TW and Lai HC: Synchronous occurrence of primary neoplasms in the uterus with squamous cell carcinoma of the cervix and adenocarcinoma of the endometrium. Taiwan J Obstet Gynecol 45: 336-339, 2006.

7. Takeda T, Sagae S, Koizumi M, Terasawa K, Ishioka S, Takashima S and Kudo R: Multiple primary malignancies in patients with gynecologic cancer. Int J Gynecol Cancer 5: 34-39, 1995.

8. Benito V, Beltrán L, Andújar M, Lubrano A and Falcón O: Synchronous primary carcinoma of the cervix and endometrium with early lymph node recurrence. Int J Gynaecol Obstet 103: 181-182, 2008.

9. Tong SY, Lee YS, Park JS, Bae SN, Lee JM and Namkoong SE: Clinical analysis of synchronous primary neoplasms of the female reproductive tract. Eur J Obstet Gynecol Reprod Biol 136: 78-82, 2008.

10. Ayhan A, Yalçin OT, Tuncer ZS, Gürgan T and Küçükali T: Synchronous primary malignancies of the female genital tract. Eur J Obstet Gynecol Reprod Biol 45: 63-66, 1992. 